\title{
Calculi in a Female Urethral Diverticulum
}

\author{
Ji Sung Shim, Mi Mi Oh, Jae Il Kang, Sun Tae Ahn, Du Geon Moon, Jeong Gu Lee \\ Department of Urology, Korea University School of Medicine, Seoul, Korea
}

\begin{abstract}
Urinary stones are rarely seen in the urethra and are usually encountered in men with urethral stricture or diverticulum. The case of a 52-year-old woman presented, who consulted for weak stream associated with repeated urinary infections. The diverticulum was approached via vaginal route and the extraction was successful. The patient has been well, with no dysuria, dyspareunia, incontinence for 3-month follow-ups.
\end{abstract}

Keywords: Female; Urethral diverticulum; Stone

Urethral diverticulum is commonly found in women, but the presence of urethral stone is rare. It could sometimes be found in men with tortuous urethra or with diverticulum. Because of a short urethra and the low probability of formation of bladder stones, this pathology is even much rare in women [1]. We report a case of female urethral diverticulum with stones.

\section{CASE REPORT}

A 52-year-old woman presented to our department with a 4 year history of recurrent urinary infections. The patient also complained of lower abdominal pain, dysuria and dyspareunia. Her past medical history included total abdominal hysterectomy 20 years ago because of uterine myoma. She was on no home medications. We decided to have further evaluation considering her recurrent infections.

On pelvic examination, at the step of compressing, leakage of purulent discharge from orifice was noted. Cystourethroscopy showed 2 orifices of diverticulum (Fig. 1). We performed enhanced abdomino-pelvic computed tomography (CT) scan instead of cystourethrography; because if the stone obliterates the opening, radiologic contrast cannot fill the sac and there is no clear evidence of the diverticulum [2]. The CT scan revealed diverticulum with stone formation under the pubis (Fig. 2). On admission, vital signs were all normal and laboratory tests dem- onstrated microscopic pyuria (5-9/high power field). General anesthesia was used for surgery. Before initiating the procedure, a 18 Fr Foley catheter was placed to isolate the urethra. A 3-cm incision was made in the median vaginal wall. Diverticulum was punctured by electrocautery and dissected with peri-urethral tissue. With 2-0 silk suture, punctured site of diverticulum was tagged (12, 3 and 9 o'clock) and we could retract diverticulum which contained small stones. Diverticulectomy was performed using a 3-layer closure (urethral wall, periurethral fascia, vaginal wall). After surgery the Foley catheter was kept in place for 14 days. Within the postoperative indications, intravenous antibiotic therapy with $3 \mathrm{rd}$ generation cephalosporin was added (1,000 mg every 12 hours) and continued for 24 hours to prevent recurrent or persistent urinary tract infections. An uroflowmetry was done and showed good voiding pattern (peak flow rate $24 \mathrm{~mL} / \mathrm{sec}$, voided urine volume $250 \mathrm{~mL}$ and residual urine volume $10 \mathrm{~mL}$ ) without de novo stress urinary incontinence.

\section{DISCUSSION}

Dysuria, dyspareunia and post-void dribbling are the classic symptoms suggestive of urethral diverticulum, but it frequently go undetected in women. Many clinicians do not perform further evaluation when a urethral mass is absent as the symptoms
Corresponding author: Jeong Gu Lee

Department of Urology, Korea University School of Medicine, Anam-dong 5-ga, Seongbuk-gu, Seoul 136-705, Korea

Tel: +82-2-920-5683 / Fax: +82-2-928-7864 / E-mail: jeongkl@kumc.or.kr

Submitted: December 1, 2010 / Accepted after revision: March 20, 2011
This is an Open Access article distributed under the terms of the Creative Commons Attribution Non-Commercial License (http://creativecommons.org/licenses/by-nc/3.0/) which permits unrestricted non-commercial use, distribution, and reproduction in any medium, provided the original work is properly cited. 

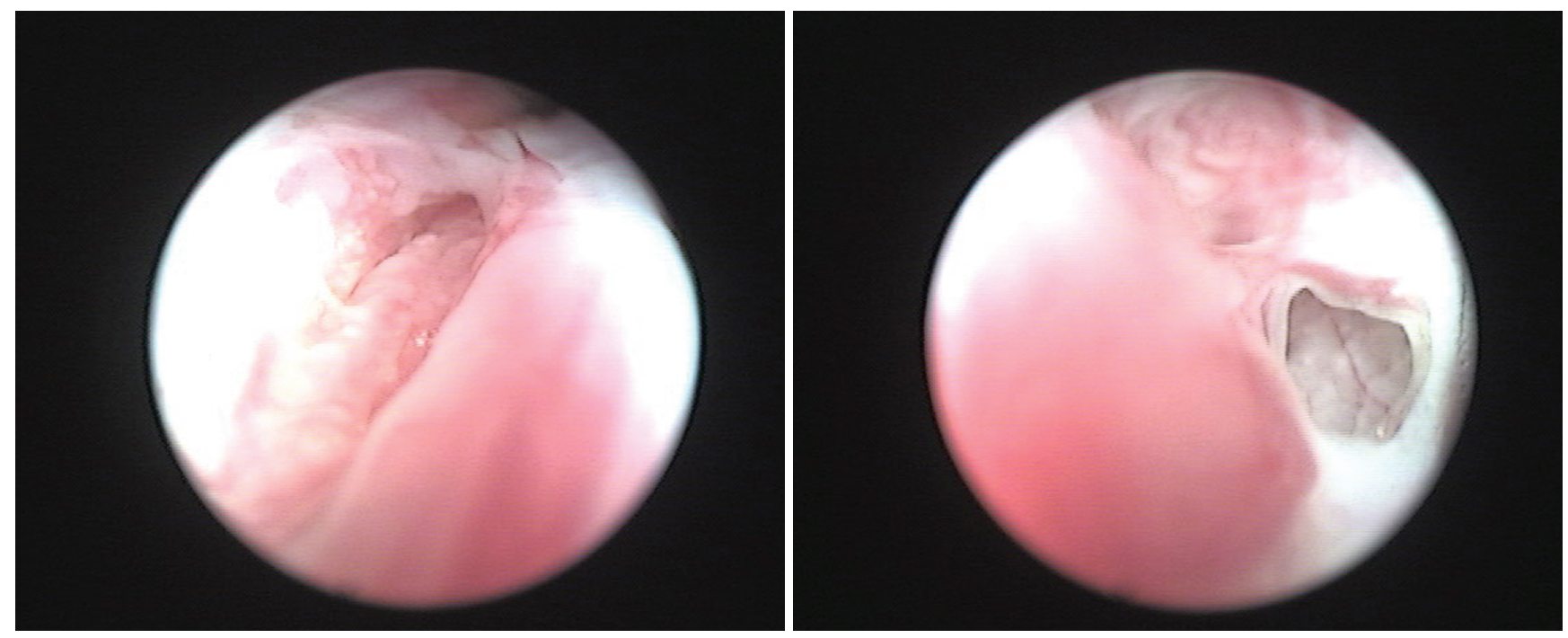

Fig. 1. Cystourethroscopy showed orifices of diverticulum.

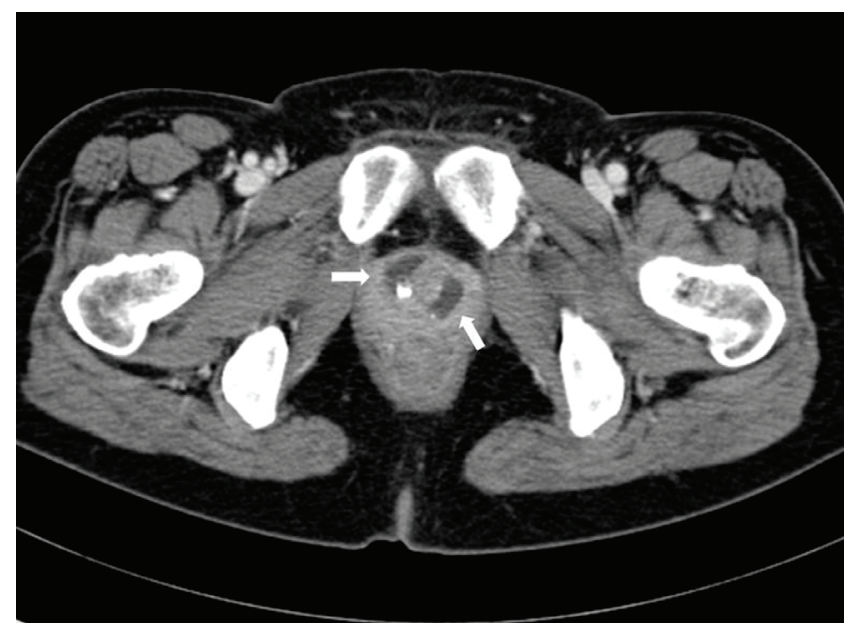

Fig. 2. Imaged by abdomino-pelvic computed tomography: two diverticulum with calculi encasing urethra (arrows).

are not specific. However, a diverticulum should be suspected in patients with recurrent urinary tract infection as well as 3 symptoms stated above because immediate diagnosis and appropriate treatment are important for this disease.

The reported incidence of urethral diverticulum in adult women is between $0.6 \%$ and $6 \%$, and associated stone formation is reported in $1.5-10 \%$ of cases [3,4]. Urethral calculi are rare and generally more common in men with urethral stricture or diverticulum. The quality of life of patients who have a diverticulum (especially with calculi) may be significantly disturbed because of complications such as mass effect, incontinence, pain, and urinary tract infection.
Any patient with lower urinary tract symptoms that have proved to be unresponsive to traditional treatment should be suspected of having an urethral diverticulum [4]. Inflammation, calculus formation and neoplastic degeneration are the main complications of untreated diverticulum [5]. Stasis of infected urine with deposition of salts and occasional mucoid desquamation of the epithelial lining is known to be the casual factor for stone formation [6]; these stones are usually composed of magnesium ammonium phosphate while migratory stones are often composed of calcium phosphate or calcium carbonate.

Urethral calculi are generally classified as native (those formed de novo in the urethra) or migratory (those formed in the bladder or kidney with secondary descent). Migrant calculi are reported to be at least 10 times more common than native calculi $[7,8]$. Most native calculi form in association with chronic stasis and urinary infection, either within a urethral diverticulum or proximal to a urethral obstruction $[9,10]$.

The single most important diagnostic indication of a calculus in the diverticulum is the finding of a palpable hard mass on the floor of the urethra [6]. Cystourethrography is important in confirming the presence of an urethral diverticulum and even the calculus within it; however, this is not always enough because of the possibility which is not fulfilled by the contrast to the sac [7]. So, CT scan or magnetic resonance imaging can be good alternative choice.

The clinical presentation of urethral calculi is variable. Specific signs and symptoms usually depend on the anatomic location of the stone. Anterior urethral stones cause dysuria and may 
be confirmed by palpation. On the other hand, posterior urethral calculi may produce pain referred to the rectum or perineum [7].

Conservative therapy offered symptomatic relief in most cases but did not prevent recurrent symptoms, so we believe that satisfactory long-term treatment of diverticulum is achieved successfully by surgical excision. Also, confirming of number, site and size of diverticulum is important before operation to prevent complications such as urethral stricture, urethro-vaginal fistula and incontinence due to injury of sphincter.

\section{CONFLICT OF INTEREST}

No potential conflict of interest relevant to this article was reported.

\section{REFERENCES}

1. Gómez Gallo A, Valdevenito Sepúlveda JP, San Martín Montes M. Giant lithiasis in a female urethral diverticulum. Eur Urol 2007;51: 556-8.
2. Lin $\mathrm{HC}, \mathrm{Wu}$ WJ, Huang $\mathrm{CH}$. A ring-shaped stone in a female urethral diverticulum: case report and review of literature. Int Urogynecol J Pelvic Floor Dysfunct 2008;19:1309-10.

3. Skyggebjerg KD. Female urethral diverticulum with calculus. Acta Obstet Gynecol Scand 1986;65:797-8.

4. Raz S. Female urology. Philadelphia: Saunders; 1983. p.378-93.

5. Aragona F, Mangano M, Artibani W, Passerini Glazel G. Stone formation in a female urethral diverticulum. Review of the literature. Int Urol Nephrol 1989;21:621-5.

6. Presman D, Rolnick D, Zumerchek J. Calculus formation within a diverticulum of the female urethra. J Urol 1964;91:376-9.

7. Larkin GL, Weber JE. Giant urethral calculus: a rare cause of acute urinary retention. J Emerg Med 1996;14:707-9.

8. Herman L, Greene LB. Diverticulum of the female urethra. J Urol 1944;52:599-610.

9. Dalens B, Vanneuville G, Vincent L, Fabre JL. Congenital polyp of the posterior urethra and vesical calculus in a boy. J Urol 1982;128: 1034-5.

10. Ginsberg P, Finkelstein LH. Urethral diverticulum with calculi: report of a case. J Am Osteopath Assoc 1983;82:588-90. 